\title{
Multifunctional nanosystems: growing sanguinity in siRNA therapy
}

\author{
This article was published in the following Dove Press journal: \\ International Journal of Nanomedicine \\ 10 April 2014 \\ Number of times this article has been viewed
}

\section{Khan Farheen Badrealam Mohammad Owais}

Molecular Immunology Laboratory, Interdisciplinary Biotechnology Unit, Aligarh Muslim University, Aligarh, Uttar Pradesh, India
Correspondence: Mohammad Owais Molecular Immunology Laboratory, Interdisciplinary Biotechnology Unit, AMU, Aligarh, India 202002

Tel +9l 57| 2720388

Fax +9| 57| 272I776

Email owais_lakhnawi@yahoo.com

\section{Dear editor}

This letter is in regards to the enlightening work published in the International Journal of Nanomedicine on multifunctional nanosystems developed for surmounting the stumbling blocks of small interfering ribonucleic acid (siRNA) therapeutics. ${ }^{1-4}$ These published studies make considerable advances toward realizing the great potential of RNA interference (RNAi)-based therapeutics. Despite the curability of the cause of diseases by rectifying altered functionalities of proteins, as of yet no technically viable and safe strategy has been established, owing to their various associated challenges. ${ }^{5}$ Albeit viral vectors are considered as the most promising strategy, mainly due to their high transduction efficiency and oligonucleotide packaging ability; however their widespread applicability has been marred due to various safety concerns. ${ }^{6}$ In this regard, nonviral vectors are very attractive, offering a safer alternative, but at a cost of lower transfection efficiency. However, with the advent of next generation nanoparticulate systems, multifunctional nanodelivery systems may bring newer hope to the RNAi therapeutic regimen.

In this arena of nanodelivery platforms for siRNA delivery, lipid-based systems (especially liposomes) shares a reputed history. In the past, cationic liposomes were the "gold standard" in vitro method for gene delivery, owing to their propensity to form efficient stable complexes with negatively charged oligonucleotides. Although, with the realization that cationic lipids are associated with various toxicity issues, neutral liposomal formulations were reconsidered as a promising systemic RNAi modulator delivery system. However, owing to their good attributes, cationic lipid formulations are still employed in research endeavours with various amendments to narrow their toxicity constraints. Recently, Chen et al ${ }^{1}$ reported the efficacy of an arginine-glycine-aspartic (RGD)-polyethylene glycol (PEG) liposomal formulation of anti-vascular endothelial growth factor (VEGF) siRNA as a promising strategy for ocular gene therapy. Reckoning with the fact that integrin receptors are overexpressed on retinal pigment epithelial cells; it has been anticipated that a carrier system fabricated with the homing ligand of integrin receptor viz Arg-Gly-Asp (RGD) motif peptides could be employed for specific delivery to retinal pigment cells. In this regard, the group made conclusive study to optimize a targeted liposomal carrier system for delivery of anti-VEGF-siRNA to retinal pigment cells to avert choroidal neovascularization. Intriguingly, optimized RGD-PEGylated liposomes were demonstrated to be a viable carrier system of siRNA to mediate efficacious knockdown of VEGF in retinal pigment epithelial cells by mediating specific targeting to retinal pigment cells embarking on the potential for gene therapy in retinopathy. ${ }^{1}$ 
Immunoliposomes, namely liposomes decorated with targeted homing antibodies, represent another development in the field and are generally fabricated by employing functionalized PEG moieties (conjugating linker) which allow coating with homing ligands, in addition to providing protection in the systemic circulation by limiting opsonization and uptake by mononuclear phagocyte system. Intriguingly, to provide a deeper insight of the influence of different conjugation derivatives on gene silencing mechanisms; Deng et $\mathrm{al}^{2}$ formulated two different anti-epidermal growth factor receptor (EGFR)-fragment antigen binding (Fab') conjugated immunoliposomal formulations (targeted liposome-polycation-DNA complex; TLPD) bearing DSPE-PEG-COOH (distearoyl-N-(3-carboxypropionoyl poly(ethylene glycol) succinyl)phosphatidylethanolamine) and DSPE-PEG-MAL (1,2-distearoyl-sn-glycero-3-phosphoethanolamine-N(maleimide poly(ethylene glycol)-2000)) ammonium salt as conjugation linkers and evaluated their physicochemical properties, namely particle size, zeta potential, stability, encapsulation efficiency, targeting ability and efficacy in mediating siRNA delivery to SMMC-7721 cells. Interestingly, both the systems appeared promising, sharing most attributes including high entrapment efficiency, improved serum stability and minor immunotoxicities. However, TLPD modified with DSPE-PEG-MAL for conjugation with anti-EGFR Fab' was more efficacious in targeting SMMC-7721 hepatocellular carcinoma cells for siRNA delivery as compared to TLPD containing DSPE-PEG-COOH. ${ }^{2}$ This study corroborated the fact that distinct conjugation linkers employed for conjugating antibodies with immunoliposomes influences the physicochemical behavior of LPD and antibody conjugation efficacy, thereby influencing their gene silencing attributes. This report will certainly help researchers in selecting linkers for formulating better targeted drug delivery systems, additionally substantiated the higher efficacy of targeted liposomes over nontargeted systems; thereby paving the way toward utilization of smart and advanced drug delivery systems via multifunctional nanosystems.

Moreover, Malhotra et $\mathrm{al}^{3}$ developed a chitosan-PEGTAT nanoformulation for the cytosolic delivery of siRNA; the system was endowed with properties to achieve cytosolic delivery owing to presence of the TAT (cell penetrating peptide), and efficiently form complexes with the negatively charged oligonucleotides due to the positively charged chitosan moieties. Moreover, the researchers addressed the toxicity issue of the cationic polymers by modifying their surfaces with the gold standard stealthing agent PEG. ${ }^{3}$ Furthermore, polyethyleneimine (PEI) has also been exploited to mediate cytoplasmic delivery of siRNA owing to its ability to enable endosomal escape via the "proton sponge" effect; however, its efficacious utilities in biological systems were limited due to its high toxicity, including proinflammatory effects. Nevertheless, various hybrid systems employing PEI and chitosan have been proposed, which not only caters their adverse effects but also bestow other desirable features. To this end, Park et $\mathrm{al}^{4}$ developed O-carboxymethyl chitosan decorated-branched PEI (OCMPEI) copolymers and appraised their suitability for the delivery of siRNA and plasmid DNA (pDNA) as well. Importantly, their system advances the delivery of oligonucleotides in in vitro and in vivo systems. ${ }^{4}$

Moreover, adding to the interest, we would like to mention that encapsulating siRNA in fusogenic nanoliposomes prepared with the plasma membrane lipid from Escherichia coli mediated the cytosolic delivery of siRNA. In this way, the system mediated efficacious downregulation of Plk-1 (pololike kinase 1) expression in in vivo systems ${ }^{5}$ and resulted in better survival of animals with liver cancer without imposing any toxicity constraints, thereby paving the way for a system with added virtues to confer potentialities to siRNA to harness their prospects in the field of molecular therapeutics.

Multifunctional nanosystems offers a promising way to navigate through multiple biological constraints, and as a result they are watched with keen interest; though there are still many daunting challenges in extrapolating these formulations into clinical settings, which still requires considerable effort from diverse sectors. Of note as these vogues persist, multifunctional nanosystems with increasing complexity and efficacy could be realized in harnessing the attribute of gene therapy in a real sense.

\section{Disclosure}

The authors report no conflicts of interest in this correspondence.

\section{References}

1. Chen CW, Yeh MK, Shiau CY, Chiang CH, Lu DW. Efficient downregulation of VEGF in retinal pigment epithelial cells by integrin ligandlabeled liposome-mediated siRNA delivery. Int $J$ Nanomedicine. 2013;8:2613-2627.

2. Deng L, Zhang Y, Ma L, et al. Comparison of antiEGFRFab' conjugated immunoliposomes modified with two different conjugation linkers for siRNa delivery in SMMC-7721 cells. Int J Nanomedicine. 2013;8:3271-3283.

3. Malhotra M, Tomaro-Duchesneau C, Saha S, Kahouli I, Prakash S. Development and characterization of chitosan-PEG-TAT nanoparticles for the intracellular delivery of siRNA. Int $J$ Nanomedicine. 2013;8:2041-2052.

4. Park SC, Nam JP, Kim YM, Kim JH, Nah JW, Jang MK. Branched polyethylenimine-grafted-carboxymethyl chitosan copolymer enhances the delivery of pDNA or siRNA in vitro and in vivo. Int J Nanomedicine. 2013;8:3663-3677. 
5. Chouhan A, Zubair S, Nadeem A, Ansari SA, Ansari MY, Mohammad O. Escheriosome-mediated cytosolic delivery of PLK1 specific siRNA: potential in treatment of liver cancer inBALB/c mice. Nanomedicine. 2013;0(0):1-14.
6. Thomas CE, Ehrhardt A, Kay MA. Progress and problems with the use of viral vectors for gene therapy. Nat Rev Genet. 2003;4(5):346-358.

\section{Publish your work in this journal}

The International Journal of Nanomedicine is an international, peerreviewed journal focusing on the application of nanotechnology in diagnostics, therapeutics, and drug delivery systems throughout the biomedical field. This journal is indexed on PubMed Central, MedLine, CAS, SciSearch $\AA$, Current Contents ${ } /$ Clinical Medicine,
Journal Citation Reports/Science Edition, EMBase, Scopus and the Elsevier Bibliographic databases. The manuscript management system is completely online and includes a very quick and fair peer-review system, which is all easy to use. Visit http://www.dovepress.com/ testimonials.php to read real quotes from published authors.

Submit your manuscript here: http://www.dovepress.com/international-journal-of-nanomedicine-journal 